\title{
Secular light curves of comets
}

\author{
Ignacio Ferrín \\ Center for Fundamental Physics, University of the Andes, \\ Merida, Venezuela \\ email: ferrin@ula.ve
}

\begin{abstract}
We present a resume of the fundamental ideas developed in the Atlas of Secular Light Curves of Comets (Ferrín, 2009).
\end{abstract}

Keywords. comets: general, $2 \mathrm{P} /$ Encke

\section{Introduction}

Recently I made some advances on the subject of the secular light curves of comets (SLCs) (Ferrín, 2005a, 2005b, 2006, 2007, 2008, 2009, from now on, papers I to VI). In particular the Atlas of Secular Light Curves of Comets (Paper VI) will appear in Planetary and Space Science, and is also available in the Astro-ph pre-print server (http://arxiv.org/ftp/arxiv/papers/0909/0909.3498.pdf). The Atlas contains 27 comets, 54 SLCs, and 70 plots. We are going to resume some of the knowledge derived in the Atlas.

\section{Secular Light Curves}

The magnitude at $\Delta, \mathrm{R}, \alpha$, is denoted by $\mathrm{m} 1(\Delta, \mathrm{R})$ for visual observations and $\mathrm{V}(\Delta, \mathrm{R}, \alpha)$ for instrumental magnitudes, where $\Delta$ is the comet-Earth distance, $\mathrm{R}$ is the Sun-comet distance, and $\alpha$ is the phase angle (Earth-comet-Sun). The brightness of the comet is presented in two plots, the Log $\mathrm{R}$ plot and the time plot. The reason to select the two plots is because they give independent and different parameters. The Log $\mathrm{R}$ plot may be reflected at $\mathrm{R}=1 \mathrm{AU}$ or may be reflected at $\mathrm{q}$, the perihelion distance (see Figure 1 ).

The 'reflected double-Log $\mathrm{R}$ ' plot presents the reduced magnitude vs Log $\mathrm{R}$ reflected at $\mathrm{R}=1 \mathrm{AU}$. Reduced means reduced to $\Delta=1 \mathrm{AU}, \mathrm{m}(1, \mathrm{R})=\mathrm{m}(\Delta, \mathrm{R})-5 \log \Delta$. In this plot time runs horizontally from left to right, although non linearly. Negative Logs indicate observations pre-perihelion. The value of the reflected double-Log $R$ diagram is that power laws on $R\left(R^{+n}\right)$ plot as straight lines. The slope 5 line at the bottom of the plot in the form of a pyramid is due to the atmosphereless nucleus (Figure 1). Additionally the inclusion of the $\mathrm{R}=1 \mathrm{AU}$ line allows the determination of the absolute magnitude by extrapolation (actually by interpolation of the pre and post-perihelion intervals).

The 'time plot' (Figure 2) presents the reduced magnitude vs time to perihelion. This is the most basic and simple plot. Negative times are pre-perihelion. The advantage of this plot is that time runs horizontally linearly from left to right thus showing the time history of the object.

\section{Envelope}

A visual observation of a comet is affected by several effects, all of which decrease the perceived brightness of the object: moon light, twilight, cirrus clouds, dirty optics (dust 
on the mirror), dirty atmosphere (pollution), low altitude (haze), excess magnification, the Delta-effect, etc. Thus it is a fundamental premise of The Atlas that the envelope of the observations defines the secular light curve, since there is no known physical effect that could increase+ the perceived brightness of a comet measured by two different observers, at the same instant of time.

\section{Parameters Measured From the Plots}

The $\log \mathrm{R}$ and the time plots provide a wealth of new information. There are $\sim 40$ parameters listed, of which $\sim 20$ are new and measured from the plots (Paper I).

Log $R$ Plots

The symbol legend for the log $\mathrm{R}$ plot can be found on the time plot (see Figures 1 and 2 ). The title of each plot identifies the comet in the new and old nomenclature system

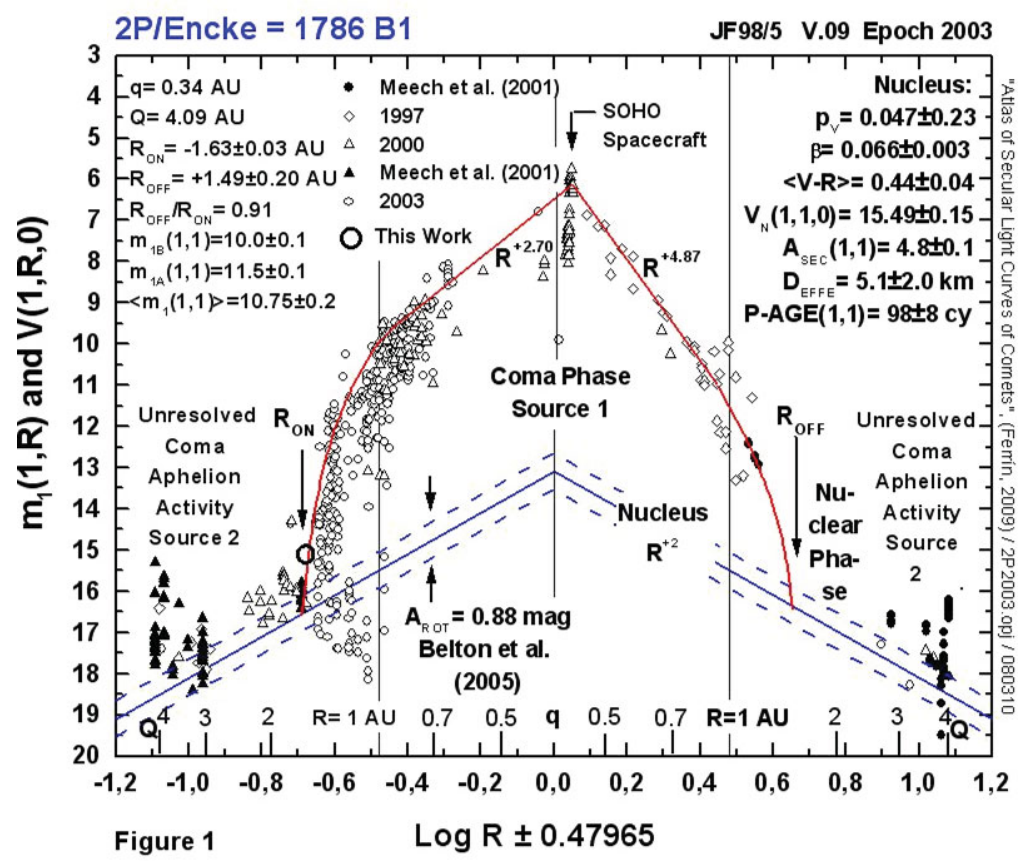

Figure 1. Secular light curve of comet 2P/Encke, Log plot. The symbols correspond to different apparitions identified in the time plot. At left and right are listed some orbital information and the parameters derived from this plot. The negative sign before logs and Rs, is a label, not a mathematical sign, and indicates only observations pre-perihelion. Since this comet has q $<1 A U, \log 0.34=0.47965$ has been subtracted before perihelion and added after perihelion to the horizontal axis to make room for the observations inside the Earth's orbit. There are three phases of the secular light curve, the nuclear phase, the coma phase and the nuclear phase again. On log plots power laws plot as straight lines, therefore the nucleus makes a pyramidal line at the bottom, since it follows a $\mathrm{R}^{+2}$ law. Most nuclear magnitudes lie inside the amplitude of the rotational light curve range $\left(\mathrm{A}_{R O T}\right)$. The turn-on and turn-off points are very sudden affairs, so it is easy to decide what is a nuclear magnitude and what observations are coma contaminated. The coma reaches a pointed sharp maximum after perihelion, and turns off with a steep descent. The secular light curve is very asymmetric with $\mathrm{R}_{O F F} / R_{O N}=0.91$. 
to allow going back to historical references. The first designation of the comet gives the first apparition (the discovery year). The label at the top right indicates if the comet belongs to the Jupiter family (JF), to the Saturn family (SF), to the Oort Cloud (OC), to the Asteroidal Belt (ABC), to the Encke type (ET), if it is a Centaur (CEN) or if it belongs to the Halley type (HT). This classification follows closely the one given by Levison (1996), except that Chiron type and Centaur are synonymous, ABC is included in his ecliptic comets and finally he did not consider the existence of Saturn family comets $(28 \mathrm{P}, 85 \mathrm{P})$. The two numbers following the family are the photometric age, P-AGE $(1,1)$ later, measured in units of comet years (cy) and the diameter measured in $\mathrm{km}$. The reason for using $\mathrm{P}-\mathrm{AGE}$ as a label here is that the definition of P-AGE is robust, as is demonstrated in Paper I.

When reading these numbers it is important to keep in mind that $\sim 95 \%$ of comets have $0<P-A G E<100 \mathrm{cy}$ and $0<D E F F E<10 \mathrm{~km}$. Any object above 100/10 or near $0 / 0$ is exceptional (very old, very large, very young, very small).

Next is the Version of the plot. All plots are identified as Version V.09 (the year of data completion). The upper left hand side of each plot gives the perihelion distance, q, the aphelion distance for that epoch, Q and Log $\mathrm{Q}$ to identify the extent of the plot. $\mathrm{Q}$ and $\mathrm{q}$ can be located at the bottom of the plots and come in units of AU. At the extreme lower right of the plots, the date line tilted $90^{\circ}$ is the last update of observations.

The Epoch label identifies the apparition that has contributed most significantly to the definition of the envelope. The importance of this label is that each apparition of the

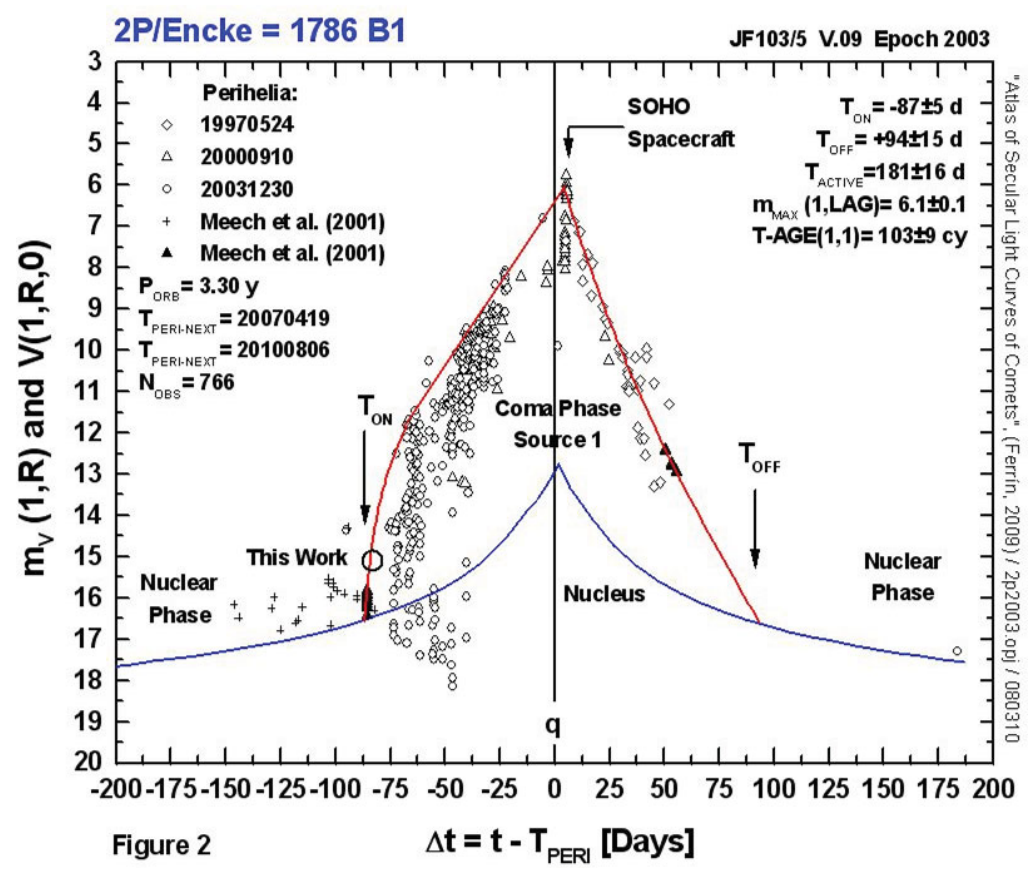

Figure 2. Secular light curve of comet $2 \mathrm{P} /$ Encke, time plot. $P_{O R B}$ is the orbital period of the comet around the sun, and the perihelia selected are indicated. The turn-off of activity is a sudden event. The comet was active for 181 days. The time-age is $T-A G E=103 \pm 9$ comet years, indicating that this is a methuselah comet. The dates are in the format YYYYMMDD. 
comet is a frame of a movie. The next apparition will have a new Epoch label. After many apparitions it will be possible to create a movie of the photometric history of the comet. The Atlas (Paper VI) contains the first frames of those movies.

Due to space limitations, we are going to describe only four parameters in the Log $\mathrm{R}$ plot, the turn on point, $\mathrm{R}_{O N}$, the turn off point, $\mathrm{R}_{O F F}$, the amplitude of the secular light curve, $\mathrm{A}_{S E C}$, and the photometric age, P-AGE (Figure 1).

1. $\mathbf{R}_{O N}[\mathbf{A U}]$. The turn-on distance of the coma. The negative sign in this parameter in $\mathrm{R}_{O N}$ and in $\log \mathrm{R}$ in the plots indicates values before perihelion but it does not mean that the $\log$ is negative. Physically $\mathrm{R}_{O N}$ corresponds to the onset of steady activity. It is the interception of the nuclear line and the coma envelope. Browsing the secular light curves of the Atlas, it can be seen that the turn-on and turn-off points are very sudden affairs. When there are enough data points these parameters can be measured easily and accurately because of the sharp change of slope. Usually $\mathrm{R}_{O N}$ takes place before perihelion, but for comets $107 \mathrm{P}$ and $133 \mathrm{P}$ it takes place after perihelion.

2. $\mathbf{R}_{O F F}[\mathbf{A U}]$ The turn-off distance of the coma, usually larger than $\mathbf{R}_{O N}$. This is the interception of the coma envelope and the nuclear line post-perihelion. $\mathrm{R}_{O F F}$ takes place after perihelion.

3. $\mathbf{A}_{S E C}(\mathbf{1 , 1})=\mathbf{V}_{N}(\mathbf{1}, \mathbf{1}, \mathbf{0})-m 1(1,1)=$ amplitude of the secular light curve above the nuclear magnitude measured at $1 \mathrm{AU}$ from the Earth, and $1 \mathrm{AU}$ from the sun. $\mathrm{A}_{S E C}(1,1)$ is a measure of the activity of the nucleus and thus of age. The value is calculated at $(1,1)$ to allow comparison to different comets. Do not confuse with $\mathrm{A}_{R O T}$ the amplitude of the rotational light curve. For comets with $\mathrm{q}<1 \mathrm{AU}$, there are two values, $\mathrm{A}_{S E C}(1,-1)$ and $\mathrm{A}_{S E C}(1,+1)$. The listed value is the mean of the pre and post-values.

4. P-AGE $(\mathbf{1}, \mathbf{1})=$ Photometric Age measured at 1 AU from the Earth, 1 AU from the sun. It is an objective of the Atlas to be able to define a parameter that measures the age of a comet solely from the secular light curves. Although it is not possible in most cases to assign an absolute physical age (exception $2 \mathrm{P} /$ Encke), it is nevertheless possible to define a parameter related to activity that ranks the comets by age. We call it P-AGE to distinguish it from a real age. It should be emphasized that P-AGE is related to the loss of volatiles as a proxy for age. The ability to order comets according to their relative ages could be a useful tool to understand a number of cometary events.

Consider the three parameters $\mathrm{A}_{S E C}, \mathrm{R}_{O N}$ and $-R_{O N}+R_{O F F}$. As a comet ages, the amplitude of the secular light curve, $\mathrm{A}_{S E C}$, must decrease. In fact $\mathrm{A}_{S E C}$ must be zero for an inert nucleus. Thus $\mathrm{A}_{S E C}$ must be related to activity and age. In the Atlas both are taken as synonymous. In fact, activity is a proxy for age. $\mathrm{R}_{O N}$ is also related to age. As the comet ages, the crust on the nucleus increases in depth, sublimating ices must recede inside the nucleus, sustained sublimation is quenched, and the comet needs to get nearer to the Sun to be activated (Yabushita \& Wada, 1988; Meech 2000). Thus $\mathrm{R}_{O N}$ decreases with age. On the other hand, $\mathrm{R}_{S U M}=-R_{O N}+R_{O F F}$ measures the total space of activity of the comet. Comets that have exhausted their $\mathrm{CO}$ and $\mathrm{CO} 2$, must get nearer to the Sun to be active. Comets whose activity is dominated by water ice become active much nearer to the Sun than $\mathrm{CO}$ or $\mathrm{CO}_{2}$ dominated comets (Meech 2000). Thus a parameter that measures age and activity at the same time, and that includes the three above quantities could be $\mathrm{A}_{S E C} *\left(-R_{O N}+R_{O F F}\right)$. This value defines the area of a rectangle in the phase space $\mathrm{A}_{S E C}$ vs $\mathrm{R}_{S U M}$. 
So defined, P-AGE would give small values for old comets and large values for new comets, inverted from what we would like. It would be interesting to scale these values to human ages. We will call these 'comet years (cy)' to reflect the fact that they have not yet been scaled to Earth's years. To calibrate the scale, we will arbitrarily set to $28 \mathrm{P} /$ Neujmin 1 an age of 100 cy. With this calibration we define P-AGE thus:

$$
P-A G E(1,1)=1440 /\left[A_{S E C}(1,1) *\left(-R_{O N}+R_{O F F}\right)\right] \text { comet years }(c y)
$$

The value of the constant has been chosen to force comet $28 \mathrm{P}$ to an age of $100 \mathrm{cy}$. Scaling to human ages may seem nä̈ve and unorthodox. However it places the comets in perspective and provides a scale for comparison. This enhances the usefulness of P-AGE, and when the evolution of $A_{S E C}, R_{O N}$ and $R_{O F F}$ with time is studied and calibrated with a suitable physical model, it will be possible to convert these values to a real physical age, thus achieving the objective we have set in this paper. This parameter classifies the secular light curves by shape, a proof of its validity (see Paper VI).

Notice that the photometric age, P-AGE, is measured at $\Delta=1 \mathrm{AU}, \mathrm{R}=1 \mathrm{AU}$, thus the correct notation is $\mathrm{P}-\mathrm{AGE}(1,1)$. This is done to be able to compare comets with different q. For comparison with the definition of absolute magnitude $\mathrm{m}(1,1)$, P-AGE $(1,1)$ could be interpreted as the absolute photometric age, and its main value is that it allows the comparison of comets with different qs.

\section{Time plots}

Due to space limitations, we are going to describe only three parameters in the Log $\mathrm{R}$ plot, the turn on point, $\mathrm{T}_{O N}$, the turn off point, $\mathrm{T}_{O F F}$ and the time-age, T-AGE $(1,1)$ (Figure 1). The symbol legend for the plots can be found on the time plot.

1. $\mathbf{T}_{O N}[$ days $]=$ the time at which the nucleus turns on. The negative sign in this parameter indicates pre-perihelion quantities. It corresponds to RON but in the time domain.

2. $\mathbf{T}_{O F F}=$ the time after perihelion at which the nucleus turns off.

3. T-AGE $(\mathbf{1}, \mathbf{1})=$ time-age. It is possible to define an age from the time plot in the same way we did for P-AGE:

$$
T-A G E(1,1)=90240 /\left[A_{S E C}(1,1) *\left(-T_{O N}+T_{O F F}\right)\right] \text { comet years }(c y)
$$

The value of the constant has be chosen to force comet $28 \mathrm{P}$ to an age of $100 \mathrm{cy}$.

\section{Example}

Due to space limitations, we can present only the SLC of one comet, 2P/Encke in 2003. It can be seen in Figures 1 and 2. See the Atlas (paper VI) for 26 additional comets.

\section{Acknowledgements}

To FUNDACITE-Mérida and CDHT-ULA for their support through several grants.

\section{References}

Ferrín, I., 2005a, Icarus, 178, 493

Ferrín, I., 2005b, ICQ, 27, 249

Ferrín, I., 2006, Icarus, 185, 523 
Ferrín, I., 2007, Icarus, 187, 326

Ferrín, I., 2008, Icarus, 197, 169

Ferrín, I., 2009, Planetary and Space Science, Paper VI, in press

Levison, H., 1999, ASP Conference Series., 107, 173

Meech, K. J., 2000, ASP Conference Series, 213, 207

Yabushita, S., \& Wada, K., 1988, EMP, 40, 303 